\title{
Absolute Velocities Are Unmeasurable: Response to Middleton and Murgueitio Ramírez
}

\author{
Caspar Jacobs*
}

November 3, 2020

\begin{abstract}
In this journal, Middleton and Murgueitio Ramírez argue that absolute velocity is measurable, contrary to the received wisdom. Specifically, they claim that 'there exists at least one reasonable analysis of measurement according to which the speedometer in [a world called 'the Basic World'] measures the absolute velocity of the car'. In this note I critically respond to this claim: the analysis of measurement that Middleton and Murgueitio Ramírez propose is neither reasonable, nor does it entail that absolute velocities are measurable.
\end{abstract}

Keywords: absolute velocity, symmetry, measurement, Newtonian mechanics

${ }^{*}$ University of Oxford, Magdalen College Oxford, UK OX1 4AU, caspar.jacobs@philosophy.ox.ac.uk 
It is orthodoxy that the values of quantities that vary under symmetries, such as absolute velocity, are unmeasurable. This wisdom has come under attack, in this journal, from Middleton and Murgueitio Ramírez (2020), whose main claim is that 'there exists at least one reasonable analysis of measurement according to which the speedometer in the Basic World measures the absolute velocity of the car' (6). ${ }^{1}$ Here, the Basic World is a Newtonian world that consists solely of a car endowed with a speedometer travelling along an infinitely long road which is at rest with respect to absolute space. I agree with Middleton and Murgueitio Ramírez (henceforth: M\&MR) that the orthodoxy pays insufficient attention to issues in metaphysics and epistemology, relying on simplistic accounts of measurement. However, I believe that their attack on the orthodoxy is unsuccessful: the analysis of measurement that M\&MR propose is neither reasonable, nor does it entail that absolute velocities are measurable. In this note I explain why.

The standard argument that absolute velocities are unmeasurable in Newtonian worlds goes as follows. ${ }^{2}$ Suppose that:

Perception-Grounded Modal Analysis. The pointer variable $P$ of a device measures a quantity $Q$ at time $t$ iff $P(t)=Q(t)$ whenever some observable conditions $C$ are satisfied.

In the actual world $W$ let $P(t)=v(t)$, where $v(t)$ denotes the absolute velocity of some particular body. Now consider a 'boosted' world $W^{*}$ in which the velocities of all material bodies are increased by some constant amount. All parties to the debate agree that since boosts are symmetries of Newtonian mechanics, $W$ and $W^{*}$ are perceptually indistinguishable. In particular, since the pointer variable is an observable, $P^{*}(t)=P(t)$ (where $P^{*}(t)$ denotes the value of the pointer variable in $\left.W^{*}\right)$. However, $v^{*}(t) \neq$ $v(t)$, since a boost transforms all velocities. Therefore, $P^{*}(t) \neq v^{*}(t)$. If it is the case that the conditions $C$ remain satisfied after a boost, the PerceptionGrounded Modal Analysis implies that $P$ does not measure $v$ : absolute velocities are unmeasurable.

As M\&MR note, the assumption that $C$ remains satisfied after a boost is crucial here. Roberts (2008) argues for this assumption on the basis that the conditions $C$ must be perceptible. Since boosts relate perceptually indistinguishable worlds, this implies that $C$ is satisfied in $w$ iff it is satisfied in $w^{*}$. But M\&MR rightly respond that Roberts' claim is false: there are

\footnotetext{
${ }^{1}$ Wallace (2019) also challenges this orthodoxy, but I will not discuss his paper here.

2 The argument presented here follows Roberts (2008). For similar arguments, see Baker (2010); Dewar (2015); Dasgupta (2016).
} 
many conditions which are required for successful measurements yet which are unobservable, such as the exact microstate of a gas. Unfortunately, however, M\&MR do not consider other arguments in favour of the assumption that $C$ is boost-invariant. For example, Roberts $(2008,164)$ argues that if $C$ is not boost-invariant, measurement is 'counterfactually fragile in the extreme': 'the proper functioning of the [measurement device] would be so sensitive to the very things they are supposed to be reliable indicators of that any change in the latter would render them unreliable'. This line seems persuasive to me, but apart from a brief discussion of a related objection in $\S 4.3, \mathrm{M} \& \mathrm{MR}$ do not mention it.

In any case, M\&MR propose a different account of measurement, which they claim is both reasonable and allows for measurements of absolute velocity:

Counterfactual Analysis. $P$ measures $Q$ at time $t$ iff (i) $P(t)=$ $Q(t)$ and, (ii) for any $x^{\prime} \neq Q(t)$ in the range of $P$, if it had been the case that $Q(t)=x^{\prime}$, then it would have been the case that $P(t)=x^{\prime}$.

In other words, $P$ must be sensitive to counterfactual variations in the value of $Q$. M\&MR argue that the Counterfactual Analysis implies that in the Basic World - which, recall, consists of a lone car travelling along an infinitely long road which is at absolute rest — the speedometer of the car measures the car's absolute velocity. (i) From the supposition that the road is at rest it follows that the relative and absolute velocity of the car are the same and so the speedometer, which normally measures a car's relative velocity with respect to the road, accurately reports its absolute velocity. (ii) Moreover, were the car to move at a different absolute velocity, then its relative velocity with respect to the road would be different too, and so the speedometer would still accurately report its absolute velocity (or so M\&MR claim - below I argue that (ii) is not satisfied in the Basic World after all).

M\&MR assert, without much argument, that it 'is clear that the Counterfactual Analysis is independently plausible' (7). I contest this claim. It is true that (i) and (ii) are both plausible necessary conditions on measurement, but this does not imply that they are also jointly sufficient. The Counterfactual Analysis bears some similarities to Nozick's (1981) 'truthtracking' account of knowledge (which M\&MR cite), so a comparison between the two is instructive. Recall that Nozick's account of knowledge has four conditions: $\mathrm{S}$ knows that $p$ iff (1) $p$ is true; (2) $\mathrm{S}$ believes that $p$; (3) if $p$ were false, $\mathrm{S}$ would not believe that $p$; and (4) if $p$ were true, $\mathrm{S}$ would believe 
that $p$. We can translate these conditions into the language of measurement as follows:

Truth-Tracking. $P$ accurately measures $Q$ iff (i) $Q(t)=P(t)$; (ii) if it were the case that $Q(t)=x^{\prime}$ for $x^{\prime} \neq Q(t)$, then it would be the case that $P(t)=x$; and (iii) if it were the case that $Q(t)=x$ for $x=Q(t)$, then it would be the case that $P(t)=x$.

Condition (i) of Truth-Tracking encompasses Nozick's (1) and (2): the device has a correct 'belief' about the value of $Q(t)$. Condition (ii) is the analogue of Nozick's (3). However, there is no analogue to Nozick's (4) in M\&MR's account of measurement. But (4) is no trivial condition: it ensures that the correctness of $S$ 's belief is not a mere accident. For example, suppose that by mistake the details of some government conspiracy have leaked and so I come to believe that the president has committed a serious crime. However, in the nearby world in which the conspiracy is covered-up, I would wrongly believe that the president has not committed any crime. Since (4) is not satisfied here, my belief fails to count as knowledge. In the case of measurement, (iii) fulfills the same role as (4), namely to ensure that $P$ 's 'belief' is not an accident. The question then is what nearby worlds there are in which the car has the same absolute velocity that it has in the basic world, that is, which worlds fulfill the antecedent of (iii)'s counterfactual conditional. These are worlds in which the road's absolute velocity is slightly different, such that the road happens to move at a constant velocity rather than remain at rest. In those worlds, the relative velocity of the car with respect to the road is not the same as its absolute velocity, and so the speedometer does not accurately report the car's absolute velocity: (iii) is not satisfied. Therefore, on a slightly more plausible account of measurement than the Counterfactual Analysis, the absolute velocity is no longer measurable in the Basic World.

Now, neither I nor M\&MR have set out clear criteria for what counts as a 'reasonable' analysis of measurement. But the fact that their account differs on crucial points from extant analyses in the literature - namely in the omission of a condition similar to (4) - gives us reason to believe that the Counterfactual Analysis is not as plausible as it at first seemed. And according to an analogue of Nozick's more plausible account, absolute velocity is unmeasurable after all. So the claim that there is a reasonable account of measurement on which absolute velocity is measurable has yet to be borne out.

Furthermore, even if M\&MR's Counterfactual Analysis is perfectly plausible, it does not support the claim that absolute velocities are measurable in 
the Basic World. Specificially, condition (ii) of the Counterfactual Analysis fails for absolute velocity measurements, as I will now show. The central contention of M\&MR's argument is that if the absolute velocity of the car were different, then the relative velocity between the car and road would also differ. In other words, the closest world in which the counterfactual $Q(t)=x^{\prime}$ is true is one in which the car moves at a different relative velocity $x^{\prime}$ with respect to the road (which remains at rest). Call this world the Relative World. But it may seem that the closest world is rather the one in which all velocities are boosted by a constant factor, i.e. the Boosted World. After all, the Boosted World is empirically equivalent to the Basic World. But in the Boosted World $P(t) \neq Q(t)$, so if the Boosted World is closest to the Basic World then the Counterfactual Analysis entails that absolute velocities are not measurable after all. M\&MR call this the 'Central Objection' and offer two responses. I will first reply to these responses and then offer some positive reasons to believe that the Boosted World is the closer than the Relative World, drawing on Lewis' (1979) account of counterfactuals.

M\&MR's first response is that scientists believe that the Relative World is closest to the Basic World. This is borne out by the way in which scientists generally evaluate counterfactuals. For example, when scientists consider the closest world in which some particular gas has a different temperature they don't usually imagine a world in which all temperatures are different. However, if we are to defer to scientific practice we surely ought to take into account the fact that most scientists believe that absolute velocity does not exist: this is the lesson from the equivalence of inertial frames. But if this is true, what do scientists even mean when they consider a world in which some object has a different velocity? It seems to me that scientists must refer to a world in which that object has a different relative velocity with respect to some salient frame of reference. I am happy to concede that the Relative World is the most nearby world in which the relative velocity between the car and the road is different, but this is simply not the counterfactual that we are interested in. In order to know how scientists evaluate counterfactuals that explicitly concern absolute velocity, at the very least more evidence is required. So the first response to the Central Objection fails.

Their second response is that we ought to evaluate counterfactuals with respect to similar states in the past. In particular, suppose that at some previous time $t_{0}$ the car had a different velocity $x^{\prime}$. M\&MR then claim that we ought to evaluate the counterfactual that $Q(t)=x^{\prime}$ by comparison with the state of affairs at $t_{0}$. However, it is not invariably the case that the worlds most similar to the actual world at time $t$ are also those that are 
most similar to the world at $t_{0}$. For a counterexample, consider a world similar to the Basic World but with two cars: Car A has always travelled at a speed of $90 \mathrm{~km} / \mathrm{h}$, while Car B travels at a speed of $30 \mathrm{~km} / \mathrm{h}$ at time $t$, but travelled at a speed of $100 \mathrm{~km} / \mathrm{h}$ at an earlier time $t_{0}$. Consider the counterfactual claim that exactly one of the two cars travelled at a velocity of $100 \mathrm{~km} / \mathrm{h}$ at time $t$. If M\&MR are right, the closest world in which this claim is true is one in which Car B travels at $100 \mathrm{~km} / \mathrm{h}$, since that car has had that same speed at $t_{0}$. However, in fact the closest world is one in which Car A travels at $100 \mathrm{~km} / \mathrm{h}$ at time $t$ : in that world, the state of affairs at time $t$ is only slightly different, since Car A has a slightly different velocity than it actually has, while in the first world the state of affairs at time $t$ is very different, since Car B in that world has a much higher velocity than it actually has. Therefore, past states of the world are not always a good guide towards closeness with respect to the present state, and so M\&MR's second response fails.

Moreover, there are positive reasons to believe that the Boosted World is closer to the Basic World than the Relative World. Specifically, Lewis (1979) argues that when we evaluate counterfactuals it is important to 'maximize the spatiotemporal region throughout which perfect match of particular fact prevails'. Now, the Relative World certainly alters matters of fact throughout some reasonably large region of space, namely the tube-shaped region carved out by the trajectory of the car. Does the Boosted World lead to a better or a worse match of particular matters of fact? This is a subtle question. On the one hand, a boost alters both the trajectory of the car and that of the road, so it seems as if the Boosted World differs even more from the Basic World than the Relative World. However, we can only evaluate whether two worlds agree on the matters of fact in some particular region once we have a way of identifying regions of space across those worlds. On this point, Butterfield (1989) has convincingly argued that the counterpart of a trajectory in the unboosted world is the boosted trajectory in the Boosted World. In other words, the counterpart of the region carved out by the car's trajectory in the Basic World is just the region carved out by the car's trajectory in the Boosted World, and likewise for the road. But this means that the Basic World and the Boosted World form a perfect match of particular facts throughout spacetime. By these standards, then, the Boosted World is much more similar to the Basic World than the Relative World, as the Central Objection contends.

An example may further illustrate the ways in which the Boosted World is closer to the Basic World. Suppose that at some point on the road stands a wall. In the Basic World, the car comes to a gentle stop when it hits 
the wall; hence so it does in the Boosted World. But suppose that in the Relative World the car's velocity is sufficiently high that it crashes through the wall. In the Relative World a noise is heard; bricks are launched far into space; the universe's entropy increases. There is a clear sense in which this world is less similar to the Basic World than the Boosted World, in which the wall remains intact. If this is indeed the case, then the Central Objection to M\&MR's argument succeeds and so their Counterfactual Analysis does not imply that absolute velocity is measurable in the Basic World after all.

Despite these objections, I believe that M\&MR are right that 'the debate over the measurability of symmetry-variant properties is more unsettled than previously supposed' (1). It is true that the analyses of measurement in the literature are rather unsophisticated, even though subtle issues of modality and epistemology matter a great deal. However, ultimately it seems that even a more sopisticated account of measurement supports the orthodoxy: absolute velocities are and remain unmeasurable.

\section{References}

Baker, D. J. 2010. Symmetry and the Metaphysics of Physics. Philosophy Compass 5(12): 1157-1166.

Butterfield, J. 1989. The Hole Truth. The British Journal for the Philosophy of Science 40(1): 1-28.

Dasgupta, S. 2016. Symmetry as an Epistemic Notion (Twice Over). The British Journal for the Philosophy of Science 67(3): 837-878.

Dewar, N. 2015. Symmetries and the Philosophy of Language. Studies in History and Philosophy of Science Part B: Studies in History and Philosophy of Modern Physics 52(Part B): 317-327.

Lewis, D. 1979. Counterfactual Dependence and Time's Arrow. Nô̂s 13(4): 455476.

Middleton, B. and S. M. Murgueitio Ramírez. 2020. Measuring Absolute Velocity. Australasian Journal of Philosophy 0(0): 1-11.

Nozick, R. 1981. Philosophical Explanations (Reprint Edition ed.). Cambridge, Mass.: Harvard University Press.

Roberts, J. T. 2008. A Puzzle about Laws, Symmetries and Measurability. The British Journal for the Philosophy of Science 59(2): 143-168.

Wallace, D. 2019. Observability, redundancy and modality for dynamical symmetry transformations. http://philsci-archive.pitt.edu/16622/. 